ISSN 0103-5150

Fisioter. Mov., Curitiba, v. 24, n. 3, p. 567-578, jul./set. 2011 Licenciado sob uma Licença Creative Commons

\title{
Teste de força de preensão manual: análise metodológica e dados normativos em atletas
}

\author{
Test of hand grip strength: a methodological analysis \\ and normative data in athletes
}

\author{
Alex de Andrade Fernandes ${ }^{[\mathrm{a}]}$, João Carlos Bouzas Marins ${ }^{[\mathrm{b}]}$ \\ [a] Universidade Federal de Viçosa, Departamento de Educação Física, Laboratório de Performance Humana (LAPEH), Minas \\ gerais, MG - Brasil, e-mail: alex.andrade@ufv.br \\ [b] Universidade Federal de Viçosa, Departamento de Educação Física, Laboratório de Performance Humana (LAPEH), Minas \\ gerais, MG - Brasil, e-mail: jcbouzas@ufv.br
}

\section{Resumo}

Introdução: Diferentes modalidades esportivas utilizam as mãos como importante seguimento corporal envolvido para o desempenho. Entre essas modalidades destacam-se judô, tênis, vela, remo, boxe, levantamento de peso e outras. A força de preensão manual é exigida nessas modalidades específicas, em que o nível de força gerado pode ser o diferencial no desempenho final. A dinamometria manual é um valioso instrumento a ser utilizado na detecção do talento esportivo, no controle do treinamento e na avaliação de recuperação de lesão. Objetivos: 0 objetivo deste estudo é fazer uma revisão sobre os principais aspectos metodológicos envolvidos na mensuração da força de preensão manual, utilizando o dinamômetro Jamar ${ }^{\circledR}$, identificando os aspectos relacionados à validade, confiabilidade, precisão, posição para realização do teste, posição da alça, instruções, número de medidas, período de descanso entre as tentativas, duração do tempo de contração, aquecimento pré-teste. Materiais e métodos: Foi realizado um levantamento para compor uma base de dados de diferentes modalidades olímpicas: coletivas e individuais. Foi realizada uma pesquisa eletrônica da literatura utilizando as bases de dados MEDLINE, SciELO e LILACS, sendo utilizados os seguintes descritores: força de preensão manual, dinamometria manual e, em inglês, hand, grip, strength, dynamometer, Jamar, norms e reference values. Conclusão: Os valores de força de preensão manual apresentados pelos atletas diferem de acordo com a modalidade, sexo, peso corporal, nível do atleta, idade e tipo de 
treinamento. A força de preensão manual deve estar presente nas baterias de testes para identificação de possíveis talentos esportivos.

Palavras-chave: Força da mão. Dinamômetro de força muscular. Esportes.

\section{Abstract}

Introduction: There are different sports that use the hand as a major body segment involved in performance: judo, tennis, sailing, rowing, boxing, weightlifting and others. Handgrip strength is required in sports where the level of force generated can be the difference in the final performance. Manual dynamometry is a valuable tool that can be used in the detection of sporting talents, training control and in the assessment of injury recovery. Objectives: The aim of this study is to review the main methodological aspects involved in the measurement of hand grip strength, using the Jamar ${ }^{T M}$ dynamometer and identifying the features of validity, reliability, precision, position for doing the test, handle position, instructions, number of measures, rest period between attempts, duration of contraction, and pre-test heating. Materials and methods: An electronic search of the literature available on the area was carried out, using the databases of MEDLINE, SciELO and LILACS. The keywords used were: handgrip strength, manual dynomometry, hand, grip, strength, dynamometer, Jamar, norms and reference values. Conclusion: The values of handgrip strength displayed by the athletes differ according to sport, sex, body weight, level of the athlete, age and type of training. Handgrip strength should be present in the batteries of tests to identify potential sporting talents.

Keywords: Hand strength. Muscle strength dynamometer. Sports.

\section{Introdução}

Diferentes modalidades esportivas utilizam as mãos como importante seguimento corporal envolvido para o desempenho $(1,2)$. Entre essas modalidades destacam-se: judô, tênis, vela, remo, boxe, levantamento de peso e outras. Em diferentes momentos, durante a prática desses esportes, a união de habilidade, força e resistência muscular é fundamental para o sucesso (2).

A força em suas mais variadas formas de manifestação é relevante para atletas que buscam seu máximo desempenho, podendo ser dividida em força muscular máxima (isométrica e dinâmica), potência muscular e resistência muscular localizada (3). A força muscular pode ser influenciada por elementos internos e externos. Entre os elementos internos destacam-se: a secção transversa da fibra muscular, o número de fibras musculares, a coordenação, a velocidade de contração das fibras musculares, o gênero, o tipo de fibra muscular e a idade $(4,5)$. Já entre os elementos externos se enquadram: hora do dia, método de treinamento, motivação, nutrição, doping, entre outros fatores $(6,7)$.

Para determinação da força manual é importante que uma avaliação seja objetiva, validada e reprodu- tível para ser realizada, utilizando-se de instrumento confiável, que permita ao profissional responsável pela realização do teste alcançar suas conclusões.

A força de preensão manual é exigida em modalidades específicas, em que o nível de força gerado pode ser o diferencial entre a vitória e a derrota (811). Assim, a dinamometria manual se destaca, sendo um valioso instrumento a ser utilizado na detecção do talento esportivo (7). Portanto, esse método de avaliação da força deve estar sempre presente em baterias de testes físicos aplicados a diferentes modalidades olímpicas.

Nesse sentido, diferentes instrumentos foram projetados para mensurar a força de preensão manual e são classificados em quatro categorias básicas: hidráulica, pneumático, mecânico e extensômetros. Desenvolvido por Bechtol no ano de 1954, o dinamômetro Jamar ${ }^{\circledR}$ consiste em um sistema hidráulico de aferição, sendo considerado o instrumento mais aceito para avaliar a força de preensão manual, por ser relativamente simples, fornecer leitura rápida e direta, além de sua possível utilização em diferentes campos de pesquisa. Esse instrumento é recomendado pela American Society of Hand Therapists (ASHT) para medir a força de preensão manual, sendo considerado o "padrão ouro" para avaliação da força manual (12). 
Uma série de estudos foram realizados empregando a dinamometria manual em modalidades esportivas olímpicas, como, por exemplo, em tênis de mesa (13), tênis (14), levantamento de peso (7), voleibol (15), judô (16). Esses trabalhos permitem identificar o perfil físico de diferentes modalidades olímpicas, apresentando os valores de força manual e levando em consideração os níveis dos atletas, o fator gênero, peso corporal e idade. Isso permite a elaboração de um referencial que pode ser utilizado como base para treinadores, preparadores físicos e outras pessoas da comissão técnica desenvolverem seu trabalho de diferentes maneiras, seja na recuperação de uma lesão, na detecção do talento esportivo, na avaliação do estado físico do atleta ou no controle do treinamento.

Assim, o objetivo deste estudo é fazer uma revisão da literatura sobre os principais aspectos metodológicos envolvidos na mensuração da força de preensão manual utilizando o dinamômetro Jamar ${ }^{\circledR}$, bem como levantar uma base de dados com valores de referência em diferentes modalidades olímpicas: coletivas e individuais.

\section{Materiais e métodos}

Foi realizada uma pesquisa eletrônica da literatura utilizando as bases de dados MEDLINE, SciELO e LILACS, utilizando os seguintes descritores: força de preensão manual, dinamometria manual e, em inglês, hand, grip, strength, dynamometer, Jamar, norms e reference values, todos sendo cruzados com o nome de cada modalidade olímpica, em inglês e em português. Os estudos analisados deveriam atender aos seguintes critérios de inclusão: publicação compreendida entre 1980 e abril de 2010.

Os pontos centrais de estudo foram identificar os aspectos relacionados à validade, confiabilidade, precisão, posição para realização do teste, posição da alça, instruções, número de medidas, período de descanso entre as tentativas, duração do tempo de contração, aquecimento pré-teste.

Também foi realizado um levantamento para compor os valores de referência de força de preensão manual em grupos de modalidades esportivas de lutas, modalidades coletivas e individuais. Foram tomados como base os resultados apresentados por uma coletiva de autores, em atletas de alto nível, visando a estabelecer valores de referência para cada modalidade.
Validade

Validade é a pertinência ou adequação de determinado método (teste, aparelho) para informar sobre um fenômeno de interesse (12). Os instrumentos hidráulicos são sistemas fechados e o registro da força é feito em quilogramas ou libras de força. Nessa categoria se enquadra o dinamômetro Jamar ${ }^{\circledR}$ (Asimow Engenharia, Santa Fe Springs, CA, EUA), que mede a força de preensão manual, contando com alças que podem ser ajustadas em cinco posições diferentes. Esse dispositivo é o mais divulgado e recomendado para avaliação da força de preensão manual $(12,17,18)$.

O dinamômetro Jamar ${ }^{\circledR}$ é um instrumento válido para a mensuração da força de preensão manual e adequado para determinar o nível de esforço exercido $(12,19)$.

\section{Confiabilidade}

A confiabilidade de um instrumento ou teste é determinada pela concordância dos resultados fornecidos por diferentes examinadores (confiabilidade interexaminadores) ou em momentos distintos (confiabilidade intraexaminadores ou teste-reteste). Segundo Shechtman et al. (12), a confiabilidade é determinada pelo cálculo estatístico de coeficientes de correlação. Assim, Mathiowetz et al. (17) e Bohannon et al. (20) testaram sua confiabilidade e chegaram à conclusão de que o dinamômetro Jamar ${ }^{\circledR}$ é um instrumento confiável, tanto para ser administrado por examinadores diferentes $(r \geq 0,97)$ quanto em momentos distintos ( $r \geq 0,95)$, garantindo uma documentação objetiva dos resultados.

\section{Precisão}

A precisão é a necessidade de verificar e manter a calibração de um instrumento. Esse fator tem sido destacado por alguns autores $(12,21)$. Esses estudos apontam que o dinamômetro Jamar ${ }^{\circledR}$ é um instrumento preciso para avaliação da força manual.

Shechtman et al. (12) destacam que o dinamômetro Jamar ${ }^{\circledR}$ obteve a mais alta precisão de calibração de vários instrumentos testados ( $\pm 3 \%$ ), indicando assim ser um instrumento seguro na sua calibração e precisão. 
Recomendações metodológicas para o teste de força de preensão manual

Para uma correta aplicação do teste de dinamometria manual é necessário seguir um conjunto de recomendações, sendo elas: posição para realização do teste, instruções, número de medidas, período de descanso entre as tentativas, duração do tempo de contração, aquecimento pré-teste e posição da alça. A seguir serão apresentadas com mais detalhes cada uma dessas recomendações.

a) Posição para realização do teste

A posição aprovada pela American Society of Hand Therapists (ASHT) é utilizada em diferentes estudos $(12,18,22)$, sendo considerada o "padrão ouro" para realização do teste. A posição para a avaliação da força de preensão manual que a ASHT recomenda é que o avaliado deva estar confortavelmente sentado, posicionado com o ombro levemente aduzido, o cotovelo fletido a $90^{\circ}$, o antebraço em posição neutra e, por fim, a posição do punho pode variar de $0^{\circ}$ a $30^{\circ}$ de extensão. No estudo de Shyam Kumar et al. (23), em que o objetivo era avaliar a força de preensão manual em diferentes posições do cotovelo (flexão $90^{\circ}$ e estendido), indicou que não há diferença significativa entre as duas posições. Concluindo que em esportes de raquete a posição ideal para avaliação seria com o cotovelo estendido.

Apesar desse resultado, é fundamental não utilizar variações na posição do teste, uma vez que sua alteração pode influenciar significativamente os resultados obtidos (24). Portanto, deve ser realizado um esforço para manter coerência e padronização nos procedimentos do teste, a fim de obtermos uniformidade nos resultados obtidos. Dessa forma, a recomendação da posição para realização do teste de preensão manual deve ser a preconizada pela ASHT.

\section{b) Posição da alça}

A ASHT recomenda que se utilize a segunda posição da manopla do dinamômetro Jamar ${ }^{\circledR}$, considerando a posição da alça a mais eficiente para realização do teste de preensão manual (12). Já Ashford et al. (25) recomendam que a terceira posição da alça seja utilizada para homens e a segunda para mulheres, porque os maiores valores de força de preensão manual registrados foram alcançados quando a manopla estava ajustada nessas posições.

No estudo de Boadella et al. (26), a posição da alça era selecionada pelo próprio avaliado, o que gerou um aumento significativo da força quando comparouse às posições previamente selecionadas, que seguem a recomendação da ASHT.

Aconselha-se que se façam tentativas exploratórias prévias, a fim de identificar qual posição da alça é a mais confortável, pois sofre influência diretamente do tamanho da mão do avaliado, sendo necessário, assim, o ajuste para homogeneizar futuras avaliações.

c) Instruções

Um protocolo de teste padronizado, com instruções consistentes para sua realização, pode minimizar erros e promover a confiabilidade da mensuração. As instruções específicas são importantes, assim também como o volume em que são transmitidas. Johansson et al. (27) encontraram diferença significativa entre o volume de um comando verbal e a força de contrações isométricas, em que o aumento do volume resultou em aumento força. No estudo de Shechtman et al. (12), todas as instruções para realização do teste transmitidas aos avaliados foram padronizadas, com o objetivo de evitar erros na coleta dos dados.

É importante uniformizar a forma com que as informações são transmitidas aos avaliados, assim como utilizar o mesmo tom de voz nas instruções em cada realização do teste, sem que nenhum tipo de incentivo seja aplicado ao avaliado.

\section{d) Número de medidas}

O método mais utilizado para registro da força de preensão manual máxima é a média de três medidas $(20,28)$. Variações desse método têm sido estudadas, como, por exemplo, uma medida, o melhor de duas ou três medidas $(22,29,30)$.

Dessa forma, quando avaliamos atletas, a realização de apenas uma medida, ou o registro da média de duas ou três medidas, parece não ser recomendado, sendo interessante que seja computado o maior valor registrado entre as três medidas realizadas de forma alternada em cada braço. 
e) Períodos de descanso entre as tentativas

Mathiowetz (31) avaliou os efeitos da fadiga durante a mensuração da força de preensão manual usando o método de três medidas com intervalo de 15 segundos entre elas. Participaram do estudo indivíduos normais e pacientes em programa de reabilitação. 0 autor não encontrou nenhuma diferença significativa entre as três tentativas dos dois grupos, indicando que esse tempo de recuperação seja suficiente para evitar uma possível fadiga muscular, não afetando os resultados apresentados. Assim, não é necessário estender o tempo de teste com longos períodos de descanso porque as diferenças de medidas são muito pequenas.

No estudo de Watanabe et al. (32), é recomendado um período de descanso de 60 segundos entre as medidas do teste de força de preensão manual. Entretanto, Trossman et al. (33) investigaram o efeito de períodos de descanso entre cinco medidas, não encontrando diferença significativa entre os períodos de descanso de 60s, 30s, e 15s. Assim, pode-se considerar que o tempo ideal de descanso para realização de uma nova medida seja por volta de 15 s, tendo em vista que será suficiente para restaurar os estoques de ATP-PC consumidos durante o teste. Recomendamos que as medidas sejam registradas de forma alternada entre as mãos, começando sempre pelo lado direito.

\section{f) Duração do tempo de contração}

A contração isométrica muscular pode causar aumentos potenciais na pressão arterial e frequência cardíaca (34). Os resultados indicam que, após 5 segundos de contração máxima, as elevações na pressão arterial e frequência cardíaca começam a aparecer de forma discreta, mas com o passar do tempo essas variáveis aumentam linearmente.

Recomenda-se que 3 segundos de contração máxima sejam o suficiente para registrar a leitura da força de preensão manual, sem causar dessa forma alteração significativa na pressão arterial e frequência cardíaca, o que torna o teste seguro para a maior parte da população.

\section{g) Aquecimento pré-teste}

Atividades específicas de aquecimento, na forma de preensão submáxima, resultam em aumento de força de preensão manual máxima (35). Esse efeito pode ser observado quando são comparados resultados de testes com e sem aquecimento. $\mathrm{O}$ aumento na força de preensão manual máxima é de aproximadamente um desvio-padrão, resultante do aquecimento e sendo considerado significativo.

Assim, para homogeneizar o processo de coleta de dados sugere-se que não seja realizado nenhum período de aquecimento anterior à realização do teste. Contudo, cabe destacar que o avaliado tem que receber as instruções para estar familiarizado com a forma de execução do teste.

\section{Força de preensão manual e esporte}

Estudos para identificação de possíveis talentos esportivos utilizam a força de preensão manual em suas baterias de testes $(7,14,16)$. Esses trabalhos apontam que a força de preensão manual é um elemento importante na condição física geral do atleta, que, quando relacionado com outras variáveis, indica a possível descoberta de um futuro atleta bem-sucedido.

Nas modalidades, tais como: handebol, judô, voleibol, basquetebol, tênis e tênis de mesa, as capacidades física, mental, técnica e tática são fundamentais para o desempenho do atleta. Contudo, a força de preensão manual é uma qualidade física importante nesses esportes e, quando aliada aos aspectos morfológicos e funcionais das mãos, pode ser fundamental para o bom desempenho $(1,2)$.

Kurakake et al. (6) e Degoutte et al. (8) estudaram uma particularidade que normalmente é praticada em modalidades de luta: a redução drástica do peso em períodos próximos à competição, e os resultados indicam que reduções drásticas influenciam negativamente o desempenho físico, diminuindo os valores da força de preensão manual.

Assim, o treinamento de força é fundamental para o registro de maiores níveis de força de preensão manual $(9,29)$. Em seus estudos, esses autores verificaram que o treinamento de força devidamente periodizado resulta em aumento dos valores da força manual. Szymanski et al. (36) verificaram que maiores níveis de força de preensão manual podem ser alcançados com treinamentos específicos para punho e antebraço, o que pode ser um diferencial nessas modalidades, em que a força manual é tão importante.

Quando tratamos de atletas de alto rendimento, pequenos detalhes podem fazer a diferença para que 
as vitórias aconteçam. Dessa forma, o desenvolvimento da força de preensão manual deve estar presente no planejamento de técnicos e preparadores físicos, assim como sua forma de avaliação, que deve seguir as proposições aqui apresentadas.

\section{Dados normativos}

Os dados normativos visam a apresentar valores típicos da força de preensão manual para uma determinada população. Eles podem ser usados para discriminar a força entre indivíduos, indicando aquele que se encontra dentro de uma faixa considerada adequada ou típica para a modalidade e aqueles que apresentam força inferior aos limites de referência. Essas informações são fundamentais quando utilizamos o teste de preensão manual para detecção do talento esportivo ou mesmo para indicação de um trabalho específico de força para desenvolvimento dessa qualidade física.

Uma das estratégias empregadas para determinar um possível talento esportivo é a utilização de escores padronizados z, denominada "estratégia Z" (37). Ela permite que o avaliador entenda onde um determinado escore se encontra em relação aos demais, em uma distribuição, indicando quanto acima ou abaixo da média um escore está em termos de unidades padronizadas de desvio, tendo como base um valor de referência de uma população especial, composta por atletas de alto nível, podendo esse valor ser calculado pela fórmula:

$$
\begin{aligned}
& X=\text { escore bruto ou resultado obtido } \\
& \mu=\text { média populacional } \\
& \sigma=\text { desvio-padrão } \\
& Z=(X-\mu) / \sigma
\end{aligned}
$$

Como exemplo, utilizaremos o estudo de Fry et al. (7), em que os principais atletas apresentam valor médio de força de preensão manual de " $\mu=52.5$ (kgf)" e desvio-padrão de " $\sigma=8.1$ ". Durante a realização de uma bateria de testes, um atleta apresenta um resultado de " $X=35.0$ (kgf)" e, depois dos cálculos efetuados, o valor de " $Z=2.1$ " unidades de desvio-padrão abaixo da média. 0 valor de "Z" apresentado pelo atleta indica ao avaliador que, nesse teste físico, o nível de força de preensão manual não condiz com o esperado quando comparado com os principais atletas da modalidade.
As informações normativas são usualmente descritas considerando fatores como sexo, idade e modalidade esportiva. Outro aspecto importante é considerar não apenas o valor absoluto registrado, mas o relativo pelo peso corporal ou a massa corporal magra, o que pode refinar a interpretação do resultado. Assim, a Tabela 1 destaca os valores da força de preensão manual em diferentes tipos de lutas olímpicas, a Tabela 2 destaca os valores da força de preensão manual em diferentes tipos de modalidades coletivas olímpicas e a Tabela 3 destaca os valores da força de preensão manual em diferentes tipos de modalidades olímpicas individuais.

É importante ressaltar que nem todos os trabalhos apresentados nesses quadros utilizaram o mesmo equipamento e o mesmo procedimento para coleta de dados. Isso mostra que é importante que se padronize o método e o equipamento na avaliação da força manual em atletas.

\section{Importantes pontos na interpretação dos resultados}

Há uma série de variáveis que influenciam na força de preensão manual, incluindo idade, sexo, peso, altura e região onde o teste foi realizado. Em geral, verificou-se que os homens têm maior força de preensão manual do que as mulheres $(4,5,30)$. Dessa forma, é importante que durante o processo de coleta de dados a divisão por gênero seja realizada.

A força de preensão manual tem uma relação curvilínea com a idade $(5,18)$, o que resulta em um aumento na força de preensão, com o aumento da idade, para atingir um pico entre 25-39 anos, e depois uma diminuição gradual, com o aumento da idade, provavelmente em decorrência da perda de massa muscular $(4,5,18)$. Nesse sentido, recomenda-se que as análises dos dados sejam realizadas levando em consideração a idade ou a divisão por grupos etários.

Há uma correlação positiva entre força de preensão manual, peso e estatura em indivíduos saudáveis (5). Assim, é fundamental que durante o processo de avaliação da força de preensão manual se realize a antropometria e, na análise dos dados, sua influência deva ser considerada.

Existem diferenças entre a força manual da mão dominante e não dominante. Foi relatado que a força da mão dominante é aproximadamente $10 \%$ maior do que a mão não dominante, e é referida como a regra 
Tabela 1 - Valores da força de preensão manual em diferentes tipos de lutas olímpicas

\begin{tabular}{|c|c|c|c|c|c|c|c|c|c|}
\hline M & Nív & S & INF. & $\mathbf{N}$ & I & Média DP (kgf) & Peso (kg) & $\mathrm{kgf} / \mathrm{kg}$ & $\mathbf{R}$ \\
\hline \multirow{6}{*}{ Boxe } & $\mathrm{i}$ & $M$ & - & 8 & $22.3 \pm 1.5$ & M. Dom: $58.2 \pm 6.9$ & $77.4 \pm 1.4$ & M. Dom: 0.75 & (38) \\
\hline & \multirow{5}{*}{$\mathrm{n}$} & \multirow{5}{*}{ M } & Junior & 30 & $17.6 \pm 2.9$ & $\begin{array}{l}\text { M. D: } 45.6 \pm 6.5 \\
\text { M. E: } 44.9 \pm 4.6\end{array}$ & $53.6 \pm 4.1$ & $\begin{array}{l}\text { M. D: } 0.85 \\
\text { M. E: } 0.83\end{array}$ & \multirow{5}{*}{ (39) } \\
\hline & & & Senior & 30 & $22.1 \pm 3.1$ & $\begin{array}{l}\text { M. D: } 62.7 \pm 4.8 \\
\text { M. E: } 50.1 \pm 3.8\end{array}$ & $76.7 \pm 10.9$ & $\begin{array}{l}\text { M. D: } 0.81 \\
\text { M. E: } 0.65\end{array}$ & \\
\hline & & & Peso leve & 7 & $17.4 \pm 2.6$ & $\begin{array}{l}\text { M. D: } 44.2 \pm 5.2 \\
\text { M. E: } 43.8 \pm 5.0\end{array}$ & $53.1 \pm 3.5$ & $\begin{array}{l}\text { M. D: } 0.83 \\
\text { M. E: } 0.82\end{array}$ & \\
\hline & & & Peso médio & 7 & $17.5 \pm 1.5$ & $\begin{array}{l}\text { M. D: } 50.7 \pm 3.0 \\
\text { M. E: } 48.7 \pm 4.2\end{array}$ & $63.4 \pm 3.2$ & $\begin{array}{l}\text { M. D: } 0.79 \\
\text { M. E: } 0.76\end{array}$ & \\
\hline & & & $\begin{array}{l}\text { Peso médio } \\
\text { pesado }\end{array}$ & 7 & $18.1 \pm 1.5$ & $\begin{array}{l}\text { M. D: } 52.6 \pm 5.1 \\
\text { M. E: } 50.1 \pm 5.9\end{array}$ & $74.6 \pm 5.4$ & $\begin{array}{l}\text { M. D: } 0.70 \\
\text { M. E: } 0.67\end{array}$ & \\
\hline \multirow{2}{*}{ Esgrima } & \multirow{2}{*}{$r$} & \multirow{2}{*}{ M } & Pré-treino & 8 & $12.3 \pm 0.4$ & M. Dom: $27.5 \pm 2.6$ & - & - & \multirow{2}{*}{ (9) } \\
\hline & & & Pós-treino & 8 & $12.3 \pm 0.4$ & M. Dom: $34.6 \pm 3.5$ & - & - & \\
\hline \multirow{11}{*}{ Judô } & \multirow{8}{*}{$n$} & \multirow{8}{*}{ M } & \multirow{2}{*}{$\begin{array}{l}\text { Acentuada } \\
\text { redução de } \\
\text { peso }\end{array}$} & \multirow{2}{*}{6} & \multirow{2}{*}{20.6} & $\begin{array}{l}50 \text { dias antes da } \\
\text { competição } \\
\text { M. D: } 44.8 \pm 11.0\end{array}$ & $72.5 \pm 7.0$ & M. D: 0.61 & \multirow{8}{*}{ (8) } \\
\hline & & & & & & $\begin{array}{l}4 \text { dias antes da } \\
\text { competição } \\
\text { M. D: } 38.3 \pm 6.9\end{array}$ & $68.2 \pm 6.6$ & M. D: 0.56 & \\
\hline & & & \multirow{2}{*}{$\begin{array}{l}\text { Moderada } \\
\text { redução de } \\
\text { peso }\end{array}$} & \multirow{2}{*}{8} & \multirow{2}{*}{20.1} & $\begin{array}{l}50 \text { dias antes da } \\
\text { competição } \\
\text { M. D: } 46.2 \pm 10.0\end{array}$ & $81.8 \pm 12,5$ & M. D: 0.56 & \\
\hline & & & & & & $\begin{array}{l}4 \text { dias antes da } \\
\text { competição } \\
\text { M. D: } 40.6 \pm 6,9\end{array}$ & $78.9 \pm 12,2$ & M. D: 0.51 & \\
\hline & & & \multirow{2}{*}{$\begin{array}{l}\text { Baixa redução } \\
\text { de peso }\end{array}$} & \multirow{2}{*}{8} & \multirow{2}{*}{19.7} & $\begin{array}{l}50 \text { dias antes da } \\
\text { competição } \\
\text { M. D: } 50.1 \pm 7.0\end{array}$ & $82.0 \pm 10,7$ & M. D: 0.61 & \\
\hline & & & & & & $\begin{array}{l}4 \text { dias antes da } \\
\text { competição } \\
\text { M. D: } 47.7 \pm 3.8\end{array}$ & $80,5 \pm 10.8$ & M. Da: 0.59 & \\
\hline & & & \multirow{2}{*}{ Total } & \multirow{2}{*}{22} & \multirow{2}{*}{20.1} & $\begin{array}{l}50 \text { dias antes da } \\
\text { competição } \\
\text { M. D: } 47.1 \pm 9.7\end{array}$ & $79.3 \pm 11,4$ & M. D: 0.59 & \\
\hline & & & & & & $\begin{array}{l}4 \text { dias antes da } \\
\text { competição } \\
\text { M. D: } 42.3 \pm 7.2\end{array}$ & $76,5 \pm 11,6$ & M. D: 0.55 & \\
\hline & $n$ & M & - & 8 & $25.0 \pm 5.0$ & $\begin{array}{l}\text { M. Dom: } 50.4 \pm 4.9 \\
\text { M. não dom: } 45.1 \pm 9.6\end{array}$ & - & - & $(40)$ \\
\hline & \multirow{2}{*}{$\mathrm{n}$} & \multirow{2}{*}{ M } & \multirow{2}{*}{ Realizou dieta } & \multirow{2}{*}{10} & \multirow{2}{*}{-} & $\begin{array}{l}\text { Manhã da } \\
\text { competição } \\
\text { M. Dom: } 50.4 \pm 2.5\end{array}$ & $72.1 \pm 1.4$ & M. Dom: 0.69 & \multirow{2}{*}{ (6) } \\
\hline & & & & & & $\begin{array}{l}10 \text { min após a } \\
\text { competição } \\
\text { M. Dom: } 47.4 \pm 2.9\end{array}$ & $74.5 \pm 3.4$ & M. Dom: 0.63 & \\
\hline
\end{tabular}


Tabela 1 - Valores da força de preensão manual em diferentes tipos de lutas olímpicas

(Conclusão)

\begin{tabular}{|c|c|c|c|c|c|c|c|c|c|}
\hline M & Nív & S & INF. & N & I & Média DP (kgf) & Peso (kg) & kgf/kg & $\mathbf{R}$ \\
\hline \multirow{5}{*}{ Judô } & \multirow{2}{*}{$\mathrm{n}$} & \multirow{2}{*}{ M } & \multirow{2}{*}{ Controle } & \multirow{2}{*}{10} & \multirow{2}{*}{-} & $\begin{array}{l}\text { Manhã da } \\
\text { competiçãão } \\
\text { M. Domi: } 53.9 \pm 2.4\end{array}$ & $74.7 \pm 6.7$ & M. Dom: 0.72 & \multirow{2}{*}{ (6) } \\
\hline & & & & & & $\begin{array}{l}10 \text { min após a } \\
\text { competição } \\
\text { M. Domi: } 48.6 \pm 1.9\end{array}$ & $75.1 \pm 1.6$ & M. Dom: 0.64 & \\
\hline & $n$ & $\mathrm{~F}$ & - & 45 & $18.8 \pm 3.4$ & M. Dom: $45.2 \pm 3.89$ & $72.5 \pm 15.0$ & M. Dom: 0.62 & (16) \\
\hline & \multirow{2}{*}{ ien } & \multirow{2}{*}{ M } & I & 26 & $22.8 \pm 3.4$ & $\begin{array}{l}\text { M. D: } 51 \pm 10 \\
\text { M. E: } 49 \pm 10\end{array}$ & $81.6 \pm 18.7$ & $\begin{array}{l}\text { M. D: } 0.62 \\
\text { M. E: } 0.60\end{array}$ & \multirow{2}{*}{ (41) } \\
\hline & & & $\mathrm{N}$ & 66 & $19.2 \pm 4.5$ & $\begin{array}{l}\text { M. D: } 42 \pm 11 \\
\text { M. E: } 40 \pm 10\end{array}$ & $70.4 \pm 14.7$ & $\begin{array}{l}\text { M. D: } 0.59 \\
\text { M. E: } 0.56\end{array}$ & \\
\hline \multirow{4}{*}{ Taekwondo } & \multirow{2}{*}{$n$} & M & $\begin{array}{l}\text { Com protetor } \\
\text { bucal }\end{array}$ & 11 & \multirow{2}{*}{$17.0 \pm 1.3$} & $\begin{array}{l}M+F \\
\text { M. Dom: } 34.2 \pm 7.4\end{array}$ & \multirow{2}{*}{ - } & \multirow{2}{*}{ - } & \multirow{2}{*}{ (10) } \\
\hline & & $\mathrm{F}$ & $\begin{array}{l}\text { Sem protetor } \\
\text { bucal }\end{array}$ & 10 & & $\begin{array}{l}M+F \\
\text { M. Dom: } 34.2 \pm 9.0\end{array}$ & & & \\
\hline & \multirow{2}{*}{ i } & M & - & 11 & $20.9 \pm 2.2$ & M. Dom: $56.6 \pm 7.6$ & $69.9 \pm 8.7$ & M. Dom: 0.80 & \multirow{2}{*}{ (42) } \\
\hline & & $\mathrm{F}$ & - & 12 & $18.5 \pm 2.6$ & M. Dom: $37.5 \pm 4.5$ & $62.3 \pm 7.4$ & M. Dom: 0.60 & \\
\hline
\end{tabular}

Legenda: $\mathrm{M}$ = modalidades; Nív = nível dos atletas, onde (i) internacional, (n) nacional e (r) regional; INF = informações sobre 0 trabalho; $\mathrm{N}$ = número de avaliados; $\mathrm{I}$ = idade média e desvio-padrão; $\mathrm{R}$ = referência.

Fonte: Dados da pesquisa.

Tabela 2 - Valores da força de preensão manual em diferentes tipos de modalidades coletivas

(Continua)

\begin{tabular}{|c|c|c|c|c|c|c|c|c|c|}
\hline M & Nív & S & INF. & $\mathrm{N}$ & I & Média DP (kgf) & Peso (kg) & $\mathrm{kgf} / \mathrm{kg}$ & $\bar{R}$ \\
\hline \multirow{9}{*}{ Basquetebol } & \multirow{2}{*}{$\mathrm{r}$} & M & - & 85 & \multirow{2}{*}{9 a 18} & $\begin{array}{l}\text { M.D: } 23.0 \pm 10.3 \\
\text { M. E: } 22.0 \pm 9.8\end{array}$ & \multirow{2}{*}{ - } & \multirow{2}{*}{ - } & \multirow{2}{*}{ (2) } \\
\hline & & $\mathrm{F}$ & - & 60 & & $\begin{array}{l}\text { M. D: } 18.2 \pm 4.3 \\
\text { M. E: } 18.1 \pm 4.1\end{array}$ & & & \\
\hline & \multirow{6}{*}{$\mathrm{n}$} & \multirow{6}{*}{ M } & \multirow{6}{*}{-} & 35 & 10 & M. Dom: $17.4 \pm 5.07$ & $37.2 \pm 5.4$ & M. Dom: 0.46 & \multirow{6}{*}{ (1) } \\
\hline & & & & 37 & 11 & M. Dom: $19.8 \pm 5.69$ & $38.7 \pm 5.6$ & M. Dom: 0.51 & \\
\hline & & & & 37 & 12 & M. Dom: $26.6 \pm 7.98$ & $45.5 \pm 8.6$ & M. Dom: 0.58 & \\
\hline & & & & 24 & 13 & M. Dom: $29.0 \pm 8.41$ & $50.2 \pm 8.6$ & M. Dom: 0.57 & \\
\hline & & & & 39 & $14-15$ & M. Dom: $42.2 \pm 7.95$ & $61.4 \pm 7.9$ & M. Dom: 0.68 & \\
\hline & & & & 21 & $16-17$ & M. Dom: $48.1 \pm 11.0$ & $68.4 \pm 10.6$ & M. Dom: 0.70 & \\
\hline & $\mathrm{n}$ & $\mathrm{F}$ & - & 16 & 19.9 & M. Dom: $38.8 \pm 3.8$ & $67.0 \pm 6.2$ & M. Dom: 0.57 & (43) \\
\hline \multirow{3}{*}{ Handebol } & \multirow{2}{*}{$r$} & M & - & 81 & \multirow{2}{*}{9 a 18} & $\begin{array}{l}\text { M. D: } 22.8 \pm 10.8 \\
\text { M. E: } 22.0 \pm 9.8\end{array}$ & \multirow{2}{*}{ - } & \multirow{2}{*}{ - } & \multirow{2}{*}{ (2) } \\
\hline & & $\mathrm{F}$ & - & 15 & & $\begin{array}{l}\text { M. D: } 23.1 \pm 3.2 \\
\text { M. E: } 22.8 \pm 3.1\end{array}$ & & & \\
\hline & $n$ & $\mathrm{~F}$ & - & 15 & $22.6 \pm 4.7$ & M. Dom: $45.2 \pm 3.89$ & $68.7 \pm 4.8$ & M. Dom: 0.65 & (16) \\
\hline Voleibol & $\mathrm{n}$ & M & $\begin{array}{l}\text { Jogadores } \\
\text { jovens }\end{array}$ & 234 & $24.7 \pm 4.4$ & $\begin{array}{l}\text { M. D: } 51.9 \pm 7.4 \\
\text { M. E: } 47.9 \pm 7.0\end{array}$ & $87.8 \pm 8.3$ & $\begin{array}{l}\text { M. D: } 0.59 \\
\text { M. E: } 0.54\end{array}$ & (15) \\
\hline
\end{tabular}


Tabela 2 - Valores da força de preensão manual em diferentes tipos de modalidades coletivas

\begin{tabular}{|c|c|c|c|c|c|c|c|c|c|}
\hline M & Nív & $S$ & INF. & $N$ & I & Média DP (kgf) & Peso (kg) & $\mathrm{kgf} / \mathrm{kg}$ & $\mathbf{R}$ \\
\hline Voleibol & $\mathrm{n}$ & M & $\begin{array}{l}\text { Jogadores } \\
\text { veteranos }\end{array}$ & 146 & $52.5 \pm 10.8$ & $\begin{array}{l}\text { M. D: } 54.0 \pm 8.8 \\
\text { M. E: } 50.4 \pm 8.6\end{array}$ & $88.4 \pm 10.4$ & $\begin{array}{l}\text { M. D: } 0.61 \\
\text { M. E: } 0.57\end{array}$ & (15) \\
\hline \multirow{3}{*}{ Vela } & \multirow{3}{*}{$\mathrm{i}$} & \multirow{3}{*}{ M } & Timoneiros & 10 & $30.2 \pm 8.2$ & $\begin{array}{l}\text { M. Dom: } 61.3 \pm 8.1 \\
\text { M. não dom: } 59.0 \pm 6.8\end{array}$ & $73.1 \pm 6.6$ & $\begin{array}{l}\text { M. Dom: } 0.83 \\
\text { M. não dom: } 0.80\end{array}$ & \multirow{3}{*}{ (11) } \\
\hline & & & Tripulantes & 9 & $24.7 \pm 2.7$ & $\begin{array}{l}\text { M. Dom: } 63.3 \pm 9.0 \\
\text { M. não dom: } 60.6 \pm 7.7\end{array}$ & $75.8 \pm 4.8$ & $\begin{array}{l}\text { M. Dom: } 0.83 \\
\text { M. não dom: } 0.79\end{array}$ & \\
\hline & & & Regatistas & 19 & $27.6 \pm 6.6$ & $\begin{array}{l}\text { M. Dom: } 63.1 \pm 8.3 \\
\text { M. não dom: } 59.7 \pm 7.1\end{array}$ & $74.4 \pm 5.9$ & $\begin{array}{l}\text { M. Dom: } 0.84 \\
\text { M. não dom: } 0.80\end{array}$ & \\
\hline \multirow{2}{*}{ Remo } & $n$ & M & - & 7 & $25 \pm 5.0$ & $\begin{array}{l}\text { M. Dom: } 46.2 \pm 1.2 \\
\text { M. Não Dom: } 44.1 \pm 1.1\end{array}$ & - & - & (40) \\
\hline & $\mathrm{n}$ & M & - & 28 & $25.4 \pm 1.2$ & $\begin{array}{l}\text { M. D: } 47.3 \pm 1.2 \\
\text { M. E: } 44.0 \pm 1.1\end{array}$ & $64.9 \pm 1.2$ & $\begin{array}{l}\text { M. D: } 0.79 \\
\text { M. E: } 0.67\end{array}$ & (44) \\
\hline
\end{tabular}

Legenda: $\mathrm{M}$ = modalidades; Nív = nível dos atletas, onde (i) internacional, (n) nacional e (r) regional; INF = informações sobre 0 trabalho; $\mathrm{N}$ = número de avaliados; $\mathrm{I}$ = idade média e desvio-padrão; $\mathrm{R}$ = referência.

Fonte: Dados da pesquisa.

Tabela 3 - Valores da força de preensão manual em diferentes tipos de modalidades individuais olímpicas

(Continua)

\begin{tabular}{|c|c|c|c|c|c|c|c|c|c|}
\hline$M$ & Nív & $\mathrm{S}$ & INF. & $\mathbf{N}$ & I & Média DP (kgf) & Peso (kg) & $\mathrm{kgf} / \mathrm{kg}$ & $\mathbf{R}$ \\
\hline \multirow{2}{*}{$\begin{array}{l}\text { Levantamento } \\
\text { de peso }\end{array}$} & \multirow{2}{*}{$\mathrm{n}$} & \multirow{2}{*}{ M } & $\begin{array}{l}\text { Principais } \\
\text { atletas }\end{array}$ & 20 & \multirow{2}{*}{$14.8 \pm 2.3$} & M. Dom: $52.5 \pm 8.1$ & $67.3 \pm 10.4$ & M. Dom: 0.78 & \multirow{2}{*}{ (7 } \\
\hline & & & $\begin{array}{l}\text { Outros } \\
\text { atletas }\end{array}$ & 95 & & M. Dom: $42.2 \pm 11.1$ & $62.3 \pm 16.5$ & M. Dom: 0.67 & \\
\hline \multirow{8}{*}{ Tênis } & $n$ & $M$ & - & 10 & $25.2 \pm 4.3$ & $\begin{array}{l}\text { M. Dom: } 61.4 \pm 10.8 \\
\text { M. não dom: } 53.4 \pm 9.7\end{array}$ & $72.0 \pm 8.1$ & $\begin{array}{l}\text { M. Dom: } 0.85 \\
\text { M. não dom: } 0.74\end{array}$ & (45) \\
\hline & i & $M$ & - & 12 & $13.6 \pm 1.4$ & $\begin{array}{l}\text { M. Dom: } 17.5 \pm 6.4 \\
\text { M. não dom: } 14.5 \pm 4.7\end{array}$ & $50.5 \pm 11.7$ & $\begin{array}{l}\text { M. Dom: } 0.34 \\
\text { M. não dom: } 0.28\end{array}$ & (14) \\
\hline & \multirow{2}{*}{$r$} & $M$ & - & 24 & \multirow{2}{*}{$19-24$} & $\begin{array}{l}\text { M. Dom: } 56.9 \pm 10.3 \\
\text { M. não dom: } 48.2 \pm 10.3\end{array}$ & $76.8 \pm 7.3$ & $\begin{array}{l}\text { M. Dom: } 0.74 \\
\text { M. não dom: } 0.62\end{array}$ & \multirow{2}{*}{ (46 } \\
\hline & & $\mathrm{F}$ & - & 24 & & $\begin{array}{l}\text { M. Dom: } 30.0 \pm 7.8 \\
\text { M. não dom: } 24.0 \pm 6.2\end{array}$ & $61.1 \pm 7.4$ & $\begin{array}{l}\text { M. Dom: } 0.49 \\
\text { M. não dom: } 0.39\end{array}$ & \\
\hline & $r$ & $\mathrm{~F}$ & - & 12 & $21.1 \pm 1.0$ & M. D + M. E: $31.2 \pm 4.1$ & $53.2 \pm 4.3$ & M. D+M. E: 0.58 & (43) \\
\hline & \multirow{3}{*}{$n$} & \multirow{3}{*}{$\mathrm{F}$} & $\begin{array}{l}\text { Periodização } \\
\text { não linear }\end{array}$ & 10 & $18.6 \pm 1.3$ & $\begin{array}{l}\text { M. Dom: } 36.3 \pm 4.5 \\
\text { M. não dom: } 32.1 \pm 2.2\end{array}$ & $60.8 \pm 7.8$ & $\begin{array}{l}\text { M. Dom: } 0.59 \\
\text { M. não dom: } 0.52\end{array}$ & \multirow{3}{*}{ (29) } \\
\hline & & & $\begin{array}{l}\text { Periodização } \\
\text { linear }\end{array}$ & 9 & $19.2 \pm 1.1$ & $\begin{array}{l}\text { M. Dom: } 36.9 \pm 3.5 \\
\text { M. não dom: } 34.5 \pm 2.6\end{array}$ & $60.5 \pm 7.7$ & $\begin{array}{l}\text { M. Dom: } 0.60 \\
\text { M. não dom: } 0.57\end{array}$ & \\
\hline & & & Controle & 8 & $19.3 \pm 1.6$ & $\begin{array}{l}\text { M. Dom: } 32.3 \pm 4.7 \\
\text { M. não dom: } 27.2 \pm 3.0\end{array}$ & $60.1 \pm 7.6$ & $\begin{array}{l}\text { M. Dom: } 0.53 \\
\text { M. não dom: } 0.45\end{array}$ & \\
\hline \multirow{2}{*}{$\begin{array}{l}\text { Tênis de } \\
\text { mesa }\end{array}$} & \multirow{2}{*}{$n$} & $M$ & - & 38 & $12.0 \pm 1.1$ & $\begin{array}{l}\text { M. Dom: } 27.1 \pm 5.1 \\
\text { M. não dom: } 22.4 \pm 4.1\end{array}$ & \multirow{2}{*}{-} & \multirow{2}{*}{-} & \multirow{2}{*}{ (13) } \\
\hline & & $\mathrm{F}$ & - & 25 & $11.9 \pm 0.9$ & $\begin{array}{l}\text { M. Dom: } 20.2 \pm 3.5 \\
\text { M. não dom: } 18.2 \pm 1.4\end{array}$ & & & \\
\hline
\end{tabular}


Tabela 3 - Valores da força de preensão manual em diferentes tipos de modalidades individuais olímpicas

(Conclusão)

\begin{tabular}{|c|c|c|c|c|c|c|c|c|c|}
\hline M & Nív & $S$ & INF. & $N$ & 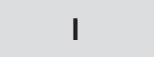 & Média DP (kgf) & Peso (kg) & $\mathrm{kgf} / \mathrm{kg}$ & $\mathbf{R}$ \\
\hline \multirow{4}{*}{ Ginástica } & \multirow{4}{*}{$r$} & \multirow{4}{*}{$F$} & Pré-menarca & \multirow{4}{*}{30} & \multirow{4}{*}{$12.3-14.5$} & $\begin{array}{l}\text { M. Dom: } 24.2 \\
\text { M. não dom: } 22.6\end{array}$ & 37.5 & $\begin{array}{l}\text { M. Dom: } 0.64 \\
\text { M. não dom: } 0.60\end{array}$ & \multirow{4}{*}{ (47) } \\
\hline & & & Pós-menarca & & & $\begin{array}{l}\text { M. Dom: } 26.1 \\
\text { M. não dom: } 25.8\end{array}$ & 43.1 & $\begin{array}{l}\text { M. Dom: } 0.60 \\
\text { M. não dom: } 0.59\end{array}$ & \\
\hline & & & Pré-menarca & & & $\begin{array}{l}\text { M. Dom: } 27.3 \\
\text { M. não Dom: } 26.9\end{array}$ & 42.4 & $\begin{array}{l}\text { M. Dom: } 0.64 \\
\text { M. não dom: } 0.63\end{array}$ & \\
\hline & & & Pós-menarca & & & $\begin{array}{l}\text { M. Dom: } 28.6 \\
\text { M. não dom: } 28.0\end{array}$ & 47.4 & $\begin{array}{l}\text { M. Dom: } 0.60 \\
\text { M. não dom: } 0.59\end{array}$ & \\
\hline
\end{tabular}

Legenda: $\mathrm{M}$ = modalidades; Nív = nível dos atletas, onde (i) internacional, (n) nacional e (r) regional; INF = informações sobre 0 trabalho; $\mathrm{N}$ = número de avaliados; I = idade média e desvio-padrão; $\mathrm{R}$ = referência.

Fonte: Dados da pesquisa.

dos 10\% (48). Crosby et al. (30) chegaram à mesma conclusão. Outros estudos constaram que a diferença entre mão dominante e não dominante é inferior a $10 \%$, ou não significativamente diferentes $(28,49)$.

Nos estudos em atletas, em que foram registrados os valores de força da mão dominante e da mão não dominante, se observa que a diferença de valores de uma mão para outra pode chegar a $20 \%$ (46). Essas diferenças parecem ser mais acentuadas nos esportes de raquete, em que a predominância de um membro sobre o outro é muito grande, o que indica também que trabalhos específicos para equilibrar esses níveis de força devem ser desenvolvidos.

Essa falta de concordância entre os estudos torna difícil tirar conclusões sobre o percentual de diferença entre a mão dominante e a mão não dominante, na força de preensão manual, e isso é obviamente influenciado pelo muitos outros fatores, tais como o trabalho e a modalidade praticada. Dessa forma, não podemos considerar nenhuma diferença percentual fixa em mãos, e a avaliação da força de preensão manual é a forma correta para verificar se existe diferença entre uma mão e outra e, se existe, qual a porcentagem de diferença.

\section{Conclusão}

O dinamômetro Jamar ${ }^{\circledR}$ é reconhecido como um instrumento padrão para medir força de preensão, apresentando bons índices de validade, confiabilidade e precisão. As instruções, o número de medidas, o período de descanso entre as tentativas, a duração do tempo de contração, o aquecimento pré-teste e a posição da alça devem ser padronizados para que haja uniformidade nas formas de avaliação.

Os valores de força de preensão manual apresentados pelos atletas se diferem de acordo com a modalidade, o sexo, o peso corporal, o nível do atleta, a idade e o tipo de treinamento. A força de preensão manual deve estar presente nas baterias de testes para identificação de possíveis talentos esportivos, principalmente nas modalidades como o judô, o boxe, a esgrima, a vela, o remo, o levantamento de peso e o tênis.

\section{Referências}

1. Visnapuu M, Jurimae T. Handgrip strength and hand dimensions in young handball and basketball players. J Strength Cond Res. 2007;21(3):923-9.

2. Barut Ç, Demirel P, Kiran S. Evaluation of hand anthropometric measurements and grip strength in basketball, volleyball and handball players. Anatomy. 2008;31(2):55-59. doi: 10.2399/ana.08.055

3. Kraemer WJ, Adams K, Cafarelli E, Dudley GA, Dooly C, Feigenbaum MS, et al. American College of Sports Medicine position stand. Progression models in resistance training for healthy adults. Med Sci Sports Exerc. 2002;34(2):364-80.

4. Vianna LC, Oliveira RB, Araujo CG. Age-related decline in handgrip strength differs according to gender. J Strength Cond Res. 2007;21(4):1310-4. 
5. Gunther CM, Burger A, Rickert M, Crispin A, Schulz CU. Grip strength in healthy caucasian adults: reference values. J Hand Surg Am. 2008;33(4):558-65.

6. Degoutte F, Jouanel P, Begue RJ, Colombier M, Lac G, Pequignot JM, et al. Food restriction, performance, biochemical, psychological, and endocrine changes in judo athletes. Int J Sports Med. 2006;27(1):9-18.

7. Fry AC, Ciroslan D, Fry MD, LeRoux CD, Schilling BK, Chiu LZ. Anthropometric and performance variables discriminating elite American junior men weightlifters. J Strength Cond Res. 2006;20(4):861-6.

8. Kurakake S, Umeda T, Nakaji S, Sugawara K, Saito K, Yamamoto Y. Changes in physical characteristics, hematological parameters and nutrients and food intake during Weight reduction in judoists. Environ Health Prev Med. 1998;3(3):152-7.

9. Tsolakis CK, Bogdanis GC, Vagenas GK, Dessypris AG. Influence of a twelve-month conditioning program on physical growth, serum hormones, and neuromuscular performance of peripubertal male fencers. J Strength Cond Res. 2006;20(4):908-14.

10. Cetin C, Kececi AD, Erdogan A, Baydar ML. Influence of custom-made mouth guards on strength, speed and anaerobic performance of taekwondo athletes. Dent Traumatol. 2009;25(3):272-6.

11. Vallejo JMB, Rosique DF, Ros EH, González-Moro IM. Fuerza máxima y resistencia muscular de agarre manual en regatistas de vela ligera de la clase tornado. Medicina de l'Esport. 2007;42(156):161-8. doi: 10.1016/S1886-6581(07)70055-5

12. Shechtman O, Gestewitz L, Kimble C. Reliability and validity of the DynEx dynamometer. J Hand Ther. 2005;18(3):339-47. doi: 10.1197/j.jht.2005.04.002

13. Tan B, Aziz AR, Chuan TK. Correlations between physiological parameters and performance in elite tenpin bowlers. J Sci Med Sport 2000;3(2):176-85. doi: 10.1016/S1440-2440(00)80079-1.

14. Girard O, Millet GP. Physical determinants of tennis performance in competitive teenage players. J Strength Cond Res. 2009;23(6):1867-72.

15. Zaccagni L, Onisto N, Gualdi-Russo E. Biological characteristics and ageing in former elite volleyball players. J Sci Med Sport. 2009;12(6):667-72.
16. Leyk D, Gorges W, Ridder D, Wunderlich M, Ruther T, Sievert A, et al. Hand-grip strength of young men, women and highly trained female athletes. Eur J Appl Physiol. 2007;99(4):415-21. doi: 10.1007/s00421006-0351-1

17. Bohannon RW, Schaubert KL. Test-retest reliability of grip-strength measures obtained over a 12-week interval from community-dwelling elders. J Hand Ther 2005;18(4):426-7. doi: 10. 1197/j.jht.2005.07.003

18. Bohannon RW, Peolsson A, Massy-Westropp N, Desrosiers J, Bear-Lehman J. Reference values for adult grip strength measured with a Jamar dynamometer: a descriptive meta-analysis. Physiotherapy. 2006; 92(1):11-15. doi: 10.1016/j.physio.2005.05.003

19. Chengalur SN, Smith GA, Nelson RC, Sadoff AM. Assessing sincerity of effort in maximal grip strength tests. Am J Phys Med Rehabil. 1990;69(3):148-53.

20. Mathiowetz V, Weber K, Volland G, Kashman N. Reliability and validity of grip and pinch strength evaluations. J Hand Surg Am. 1984;9(2):222-6.

21. Fess EE. A method for checking Jamar dynamometer calibration. J Hand Ther. 1987;1(1):28-32.

22. Coldham F, Lewis J, Lee H. The reliability of one vs. three grip trials in symptomatic and asymptomatic subjects. J Hand Ther. 2006;19(3):318-26.

23. Shyam Kumar AJ, Parmar V, Ahmed S, Kar S, Harper WM. A study of grip endurance and strengh in different elbow positions. J Orthop Traumatol 2008;9(4): 209-11.

24. Hillman TE, Nunes QM, Hornby ST, Stanga Z, Neal KR, Rowlands BJ, et al. A practical posture for hand grip dynamometry in the clinical setting. Clin Nutr. 2005;24(2):224-8.

25. Ashford RF, Nagelburg S, Adkins R. Sensitivity of the Jamar dynamometer in detecting submaximal grip effort. J Hand Surg Am. 1996;21(3):402-5. doi: 10.1016/ S0363-5023(96)80352-2

26. Boadella JM, Kuijer PP, Sluiter JK, Frings-Dresen MH. Effect of self-selected handgrip position on maximal handgrip strength. Arch Phys Med Rehabil. 2005; 86(2):328-31. doi: 10.1016/j.apmr.2004.05.003

27. Johansson CA, Kent BE, Shepard KF. Relationship between verbal command volume and magnitude of muscle contraction. Phys Ther. 1983;63(8):1260-5. 
28. Incel NA, Ceceli E, Durukan PB, Erdem HR, Yorgancioglu ZR. Grip strength: effect of hand dominance. Singapore Med J. 2002;43(5):234-7.

29. Kraemer WJ, Hakkinen K, Triplett-Mcbride NT, Fry AC, Koziris LP, Ratamess NA, et al. Physiological changes with periodized resistance training in women tennis players. Med Sci Sports Exerc. 2003;35(1):157-68.

30. Crosby CA, Wehbé MA, Mawr B. Hand strength: normative values. J Hand Surg Am. 1994;19(4):665-70.

31. Mathiowetz V. Effects of three trials on grip and pinch strength measurements. J Hand Ther. 1990;3:195-8.

32. Watanabe T, Owashi K, Kanauchi Y, Mura N, Takahara $\mathrm{M}$, Ogino T. The short-term reliability of grip strength measurement and the effects of posture and grip span. J Hand Surg Am. 2005;30(3):603-9. doi: 10.1016/ j.jhsa.2004.12.007

33. Trossman PB, Li PW. The effect of the duration of intertrial rest periods on isometric grip strength performance in young adults. OccupTher J Res. 1989;9(6): 362-78.

34. Koltyn KF, Trine MR, Stegner AJ, Tobar DA. Effect of isometric exercise on pain perception and blood pressure in men and women. Med Sci Sports Exerc. 2001;33(2):282-90.

35. Marion R, Niebuhr BR. Effect of warm-up prior to maximal grip contractions. J Hand Ther. 1992;5:143-6.

36. Szymanski DJ, McIntyre JS, Szymanski JM, Molloy JM, Madsen NH, Pascoe DD. Effect of wrist and forearm training on linear bat-end, center of percussion, and hand velocities and on time to ball contact of high school baseball players. J Strength Cond Res. 2006;20(1):231-40.

37. Böhme MTS. O tema talento esportivo na ciência do esporte. Rev Bras Cienv Mov. 2007;15(1):119-26.

38. Guidetti L, Musulin A, Baldari C. Physiological factors in middleweight boxing performance. J Sports Med Phys Fitness. 2002;42(3):309-14.

39. Khanna G, Manna I. Study of physiological profile of Indian boxers. J Sports Scien Med. 2006;5:90-8.

40. Borges NG Jr, Domenech SC, Dias JA, da Silva ACK, Sagawa Y Jr. Comparative study of maximum isometric grip strength in different sports. Rev Bras Cineantropom Desempenho Hum. 2009;11(3):292-8.
41. Franchini E, Takito MY, Kiss MAPDM, Sterkowicz S. Physical fitness and anthropometric differences between elite and non-elite judo players. Biology Sport. 2005;22(4):315-28.

42. Heller J, Peric T, Dlouha R, Kohlikova E, Melichna J, Novakova H. Physiological profiles of male and female taekwon-do (ITF) black belts. J Sports Sci. 1998;16(3):243-9.

43. Tsuji S, Tsunoda N, Yata H, Katsukawa F, Onishi S, Yamazaki H. Relation between grip strength and radial bone mineral density in young athletes. Arch Phys Med Rehabil. 1995;76:234-238.

44. Singh R, Singh JH, Sirisinghe RG. Physical and physiological profiles of Malaysian dragon boat rowers. Br J Sports Med. 1995;29(1):13-5.

45. Gojanovic B, Waeber B, Gremion G, Liaudet L, Feihl F. Bilateral symmetry of radial pulse in high-level tennis players: implications for the validity of central aortic pulse wave analysis. J Hypertens. 2009;27(8):1617-23.

46. Lucki NC, Nicolay CW. Phenotypic plasticity and functional asymmetry in response to grip forces exerted by intercollegiate tennis players. Am J Hum Biol. 2007; 19(4):566-77.

47. Haywood KM. Strength and flexibility in gymnasts before and after menarche. Br J Sports Med. 1980; 14(4):189-92.

48. Petersen P, Petrick M, Connor H, Conklin D. Grip strength and hand dominance: challenging the $10 \%$ rule. Am J Occup Ther. 1989;43(7):444-7.

49. Jarjour N, Lathrop JA, Meller TE, Roberts KS, Sopczak JM, Van Genderen KJ, et al. The 10\% rule: grip strength and hand dominance in a factory population. Work. 1997;8:83-91.
Recebido: 16/06/2010 Received: 06/16/2010

Aprovado: $14 / 05 / 2011$ Approved: 05/14/2011 